\title{
Effort and contrafreeloading
}

\author{
CYNTHIA L. FEILD, STEVE KASPER, and DENIS MITCHELL \\ University of Southern California, Los Angeles, California
}

\begin{abstract}
Three groups of rats were trained to barpress for food pellets on the left side of an operant chamber and subsequently were offered a choice between this familiar food source and a novel food source on the right side for 10 consecutive daily sessions. One group was offered a choice between barpressing for "earned" pellets from the familiar source or obtaining identical "free" pellets from the novel source. A second group was offered a choice between two earned food sources, and a third group was offered a choice between two free food sources. The results were in agreement with a neophobia-optimality model. The latter groups, which were given a choice between two equally effortful food sources, overwhelmingly preferred the familiar source. The former group, which was given a choice between the familiar earned food source and a novel free food source, preferred the novel source.
\end{abstract}

Several researchers have reported that, when offered a choice, rats prefer to work for their food as opposed to obtaining free food (Jensen, 1963; Neuringer, 1969; Carder \& Berkowitz, 1970; Singh, 1970). However, a preference for earned food is not the typical finding in this so-called "contrafreeloading" paradigm (Taylor, 1972), and studies showing a preference for earned food are difficult to replicate (see Osborne, 1977, for a review). One of the explanations of the choice behavior of rats in the contrafreeloading paradigm is the twoprocess neophobia-optimality model recently reformulated by Mitchell, Becnel, and Blue (1981). This model contends that a preference for earned or free food is determined primarily by the interaction of two sometimes competing, sometimes synergistic, behavioral propensities. Rats tend to optimize their energy expenditure for food; they are reluctant to expend energy needlessly. Rats are also neophobic; they are reluctant to eat from novel food sources (Mitchell, Scott, \& Williams, 1973).

Several studies have shown that, when neophobia is controlled by equal pretest familiarization with both the free and earned food sources, rats optimize; they are unwilling to barpress for food pellets when equally familiar, but less effortful, pellets are available (Mitchell et al., 1973, Experiment 2, 1981; Tarte \& Snyder, 1973, Experiment 4). Conversely, if the two food sources are made equally effortful, rats should, at least initially, prefer the more familiar of the two: They should manifest a neophobic avoidance of the more novel food source.

Since many researchers tacitly assume that rats in the contrafreeloading situation violate the law of least effort (optimality), it seems appropriate to determine

We want to thank Beth D. Jamieson and Robert Lennartz for their help in conducting this experiment. Correspondence and requests for reprints of the article should be sent to: Denis Mitchell, Department of Psychology, University of Southern California, Los Angeles, California 90089. what role effort plays in the choice a rat makes between the free and earned food sources. For example, Carder and Berkowitz (1970) showed that rats would violate the law of least effort if work demands were not too high. However, the neophobia-optimality model, based on data employing CRF schedules (every response reinforced), indicates that rats discriminate between free and earned food sources even under conditions of moderate differences in effortfulness (e.g., Mitchell et al., 1981). In addition to Carder and Berkowitz's basic disagreement with the matching equations of Herrnstein (1970), there has been considerable difficulty in replicating their results (cf. Atnip \& Hothersall, 1973; Hothersall, Huey, \& Thatcher, 1973; Tarte \& Vernon, 1974; Taylor, 1972).

The purpose of the present experiment was to reexamine the role of effort in the contrafreeloading paradigm. This was done by training three groups of rats to barpress for earned food and testing one group with the usual effort difference between the earned and free food sources. The other two groups were provided choice alternatives equated for effortfulness; one group was given a choice between two earned food sources, and the other was given a choice between two free food sources. By equating the effort required to obtain food from both food sources, we were able to test the prediction from the neophobia-optimality model that, if two food sources are equally effortful, rats will prefer the more familiar of the two. Comparisons of the performances of each of these groups with the performance of a group given the usual choice between free and earned food also permitted us to determine if neophobia and optimality are, in fact, codeterminants of a rat's choice behavior in this situation.

\section{METHOD}

Subjects

Twenty-four experimentally naive, male Wistar (Simonsen) 
rats approximately 120 days old at the beginning of the experiment were used as subjects.

\section{Apparatus}

Throughout the experiment, all subjects were housed in standard stainless-steel isolation cages located in an airconditioned room with overhead fluorescent lighting set for a 12-h light cycle that began at 6:00 am daily. Water was continuously available. When provided, food (Purina Rat Chow) was supplied in stainless-steel containers suspended in the back of each cage. Eight identical operant chambers (Ralph Gerbrands) programmed to deliver $0.045-\mathrm{g}$ food pellets (Noyes) on a CRF schedule were located in an adjacent room. The chambers were enclosed in sound-attenuating cubicles (Lehigh Valley) equipped with ventilating fans and overhead house lights $(7.5 \mathrm{~W})$ that remained on continuously. The operant chambers were modified such that identical reponse bars and food magazines were provided on the left and right sides. The bars, which required the same operating force (at least $0.32 \mathrm{~N}$ ), were located directly opposite to one another in the center of their respective sides. The floor-level food magazines were also positioned directly opposite to one another in the left- and right-front corners, respectively. During barpress training and earned-food familiarization, only the left bar and magazine were present; the apertures for the right bar and magazine were covered with metal plates. During testing, both sets of bars and magazines were present for all groups. Throughout the experiment, the animals were transported to and from the operant chambers in eight wooden box cages $(28.7 \times 28.0 \times 29.2 \mathrm{~cm})$ that were painted flat black and equipped with hinged wire-mesh tops. The transportation cages were mounted on a cart so that each set of eight animals could be moved from room to room simultaneously.

\section{Procedure}

All animals were food deprived for $23 \mathrm{~h}$ on each of the first 10 days prior to the onset of barpress training. Water was freely available in their home cages, and they received Purina Rat Chow during the hour that they were to be participating in the experiment. Throughout the remainder of the experiment, the subjects received $30 \mathrm{~min}$ of food while in the chamber and 30-min access to Purina Rat Chow in their home cages immediately after their group had been run. The subjects' weights fell to approximately $80 \%$ of their free-feeding weights. On Days 11 and 12 , all subjects were given magazine training, one group at a time: One pellet was delivered every $30 \mathrm{sec}$, irrespective of the subjects' responses on the bar, to a food magazine located on the left side of the chamber. Any barpresses made at this time were reinforced by the delivery of an additional pellet. Each magazinetraining session, and all other experimental sessions, lasted for $30 \mathrm{~min}$. On Days 13 and 14, all subjects were shaped for $30 \mathrm{~min}$ to barpress by successive approximations. During the next 8 days, each group was placed in the operant chambers at the same time each day and allowed to barpress for $30 \mathrm{~min}$; this constituted the training days analyzed in the results section. Each animal was always trained and tested in the same apparatus.

After training, the animals were placed by groups in the randomly preassigned test conditions. Throughout the 10 test days, Group EF (earned vs. free) was treated in the traditional manner: A barpress response was required to deliver a pellet on the left and familiar side, and 400 free pellets were offered with a nonfunctional bar (which made the usual "click" and required $0.32 \mathrm{~N}$ operating force), as a contextual cue and neophobia inhibitor, on the right side. Group EE (earned vs. earned) was offered a choice between a functional response bar on the left side of the apparatus where they had been trained, and an identical functional response bar on the right side of the chamber. Lastly, Group FF (free vs. free) was offered 400 free pellets in the magazine on the familiar left side and 400 free pellets on the unfamiliar right side. Response bars, which did not deliver pellets, were located on both sides in the free vs. free condition; these served again as contextual cues. One free, unearned pellet was dispensed into each earned-food magazine prior to the introduction of the animal.

The number of barpresses minus spillage and pellets left in the magazine, mutliplied by $0.045 \mathrm{~g}$, was recorded as the amount of earned food consumed. Free-food consumption was calculated by subtracting spillage and the weight of the pellets remaining in the free-food magazines from the original amount. Familiar-food preference was calculated as the proportion of total food consumption consisting of food from the familiar source (familiar-food consumption divided by familiar-plus unfamiliar-food consumption).

\section{RESULTS}

The results are shown in Figure 1. A repeatedmeasures analysis of variance (ANOVA) was used to determine if the three groups differed in the mean number of barpresses made during the 8 training days. There were no significant differences between the groups during training (grand means: Group $\mathrm{EF}=185.48$; Group EE $=193.36$; and Group FF $=185.36$ ). There was, however, a significant days effect $[F(7,147)=$ $18.80, \mathrm{p}<.001]$. This days effect has been observed in previous studies and is due to a gradual increase in the number of barpresses over days (Mitchell et al., 1981). Across groups, the mean for Training Day 1 was 155.33 barpresses, and that for Day 8 was 213.46 barpresses.

Similarly, a repeated-measures ANOVA was used to determine whether the percentage of food consumed on the familiar left side (where all of the animals had been trained) was significantly different among the groups over the 10 test days. As illustrated in Figure 1, the results were significant and in concordance with the predictions of the neophobia-optimality model. The ANOVA indicated a significant groups effect $[F(2,21)=$

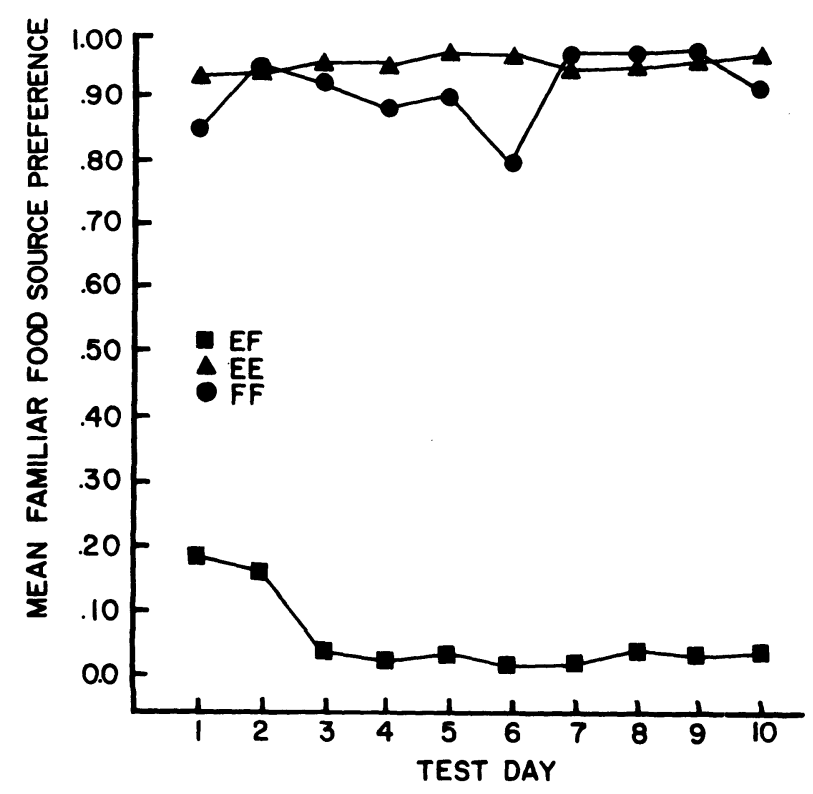

Figure 1. Mean earned-food preference for each of the three groups over the 10 test days. (Abbreviations: $E F=$ earned vs. free; $E E$ = earned vs. earned ; and $F F=$ free vs. free). 
$255.54, \mathrm{p}<.001]$. The $\mathrm{F}$ value for trial days was not significant. The group means for the percentage of food eaten from the familiar side were: Group $E F=6.19 \%$, almost all of which was due to one rat; Group $\mathrm{EE}=$ 95.61\%; and Group FF $=91.44 \%$. A Tukey's HSD post hoc analysis was performed on these means to determine which groups were significantly different from one another (HSD $\geqslant 0.4310$, using $\alpha=.05$ ): The means of Groups EF and EE were significantly different $(q=7.6780)$ from each other. Likewise, the means of Groups EF and $F F$ were significantly different $(\mathrm{q}=$ 7.319). However, the two groups with effortfulness equated (Groups EE and FF) did not differ significantly from each other in their preference for the familiar side $(q=0.3486)$.

Total pellet consumption of the three groups over the 10 test days was also analyzed using a repeatedmeasures ANOVA. The groups effect was not significant $[F(2,21)=0.96]$ (grand means: Group $E F=255.85$; Group EE $=229.15$; and Group FF $=245.80$ ). However, the days effect was significant $[F(9,189)=9.18, p<$ $.001]$, and the days $\mathrm{x}$ group interaction was significant $[F(18,189)=2.35, p<.002]$. This days $x$ group interaction reflects the increase in food consumption by groups EF and FF across tests. This gradual increase in total consumption across test days for both groups that were offered free food confirms that the free food was less effortful than the earned food; more food could be obtained during the limited 30-min test sessions by eating free food than by barpressing. The means for Day 1 and Day 10, respectively, were: Group EF $=234$ and 270 ; Group $\mathrm{EE}=231$ and 232 ; and Group $\mathrm{FF}=228$ and 275 pellets. The grand mean for the number of barpresses made on the nonfunctional bars in the freefood locations was very low (grand mean $=0.8$ ).

\section{DISCUSSION}

In addition to confirming the neophobia-optimality model's prediction that rats will prefer the more familiar of two equally effortful food sources in this paradigm, the present results are consistent with the model's assumption that neophobia and optimality are codeterminants of a rat's choice behavior in this situation. When effortfulness is equated (Groups EE and FF), the food sources are "matched" (Herrnstein, 1958, 1970). With matched effortfulness, neophobia predominates; the familiar food source is preferred because neophobia is unopposed by a competing tendency to optimize.

However, optimality can override neophobia. The rats in Group EF barpressed at a very low rate, which decelerated over the 10 test days. In addition to its compatibility with the previously cited reports that did not find a preference for earned food, the performance of Group EF demonstrates how a propensity to optimize can negate the propensity to be neophobic that was observed in Groups EE and FF. One rat in Group EF was noticeably more neophobic than the other group members, but it still barpressed for less than $30 \%$ of its food on Day 10. Most of the variability in food-source preference in Group EF was probably due to neophobia differences between and among the different animals (e.g., Mitchell, 1976). This explanation is made more compelling by Mitchell, Williams, and Sutter's (1974) finding that there is a positive correlation between preference for earned food and container neophobia.

The rats of Group EF also exhibited a repeated-testing pref- erence shift from earned to free food (Mitchell et al., 1981; Tarte \& Rasmussen, 1979; Taylor, 1972) in graphical terms, but the preference shift was not statistically significant. This observed lack of a significant repeated-testing preference shift in Group EF can also be attributed to variability in neophobia (Mitchell, 1976). The rapid shift of the animals in Group EF to the free food, and the fact that these animals and the animals in Group FF made less than one barpress per session on the nonfunctional bars in the free-food locations, further support the contention that the rats were optimizing their energy expenditures by not barpressing when it was unnecessary.

The procedure employed in the present experiment was somewhat different from the one commonly employed in this paradigm. Usually, rats are offered a choice between the food magazine of the operant chamber and a distinctive novel-food cup placed against the opposite wall. In the present study, the free- and earned-food sources were virtually identical, complete with a nonfunctional bar on the free-food side for the animals in Group EF. We used this procedure for two reasons. In the first place, we had recently found that the dimensions of the freefood cup can have a pronounced effect on a rat's preference for the "free" food (Mitchell, Fish, \& Calica, 1982). In the second place, Tarte and his colleagues (e.g., Tarte \& Snyder, 1973; Tarte \& Vernon, 1974) have routinely offered animals a choice between free- and earned-food sources consisting of identical food cups and have just as routinely found a preference for earned food.

We expected that our use of a similar procedure in which we used identical chamber-mounted magazines instead of identical food cups would produce data similar to that of Tarte and his colleagues. It did not. We not only failed to find a preference for earned food, but we also failed to find a significant amount of barpressing for earned food. (The animals in Group EF earned only $6.19 \%$ of their food across the 10 test days.) At this time, we are not certain why we failed to get a significant "contrafreeloading" effect. However, we are suspicious that the failure was due to our use of a free-food source that was not as "novel" as the usual free-food source employed in this paradigm.

It has been suggested (e.g., Barnett, 1963, p. 22) that the proclivity of rats to avoid novel foods and novel food sources is counterbalanced by a competing "sampling strategy." By initially taking only small amounts from a potentially dangerous novel food source, the animals gradually become more familiar with that source, presumably through a process of habituation. If the novel food course is safe, a rat can then optimize its foraging efficiency by comparing the novel source with its familiar sources and selecting the least effortful and/or most beneficial. This sampling strategy is probably the reason why the animals in Groups EE and FF did not entirely limit their consumption to the familiar source.

Conversely, there was some sampling of the novel food source throughout the tests by all of the animals in Groups EE and FF. One might logically assume that as the novel source become progressively more familiar across tests, the animals should have shown a shift toward selecting equal amounts from both food sources. For example, several experiments have shown that when rats are offered a choice between two comparable novel and familiar food sources, they gradually come to eat equal amounts from both (e.g., Mitchell, 1976).

However, at least one report suggests that it is not to an animal's advantage, in terms of energy costs, to continually switch from one food source to another during a limited feeding period in an operant chamber unless one food source is more economical than the other (Allison, Miller, \& Wozny, 1979). Applied to the present results, this suggestion indicates that the rats that were offered a choice between two equally effortful food sources (Groups EE and FF) did not sample the novel source extensively either because sampling was not rewarded by any gain in efficiency, as it was for Group EF, or because it was punished by a decrease in efficiency, as suggested by the results of the study by Allison and his colleagues (Allison et al., 1979). The latter interpretation might also be applicable to the finding 
of Tarte and Snyder (1972) that the more food-deprived the rat, the greater its propensity to prefer earned food in the contrafreeloading situation. Presumably, the greater the food deprivation of a rat, the greater are the energy costs of implementing its sampling strategy.

In this same vein, a rat is probably in greater danger from poisoning or predators when weak from food deprivation than when strong and satiated. Inasmuch as novel food sources are likely to be more dangerous than familiar sources, it makes sense for an animal to curtail its exposure to these sources when it is least able to deal with any harmful consequences they may portend.

\section{REFERENCES}

Allison, J., Miller, M., \&Wozny, M.(1979). Conservation in behavior. Journal of Experimental Psychology: General, 108, 4-34.

Atnip, G., \& Hothersall, D. (1973), The preference of albino rats for free or response-produced food. Bulletin of the Psychonomic Society, 2, 153-154.

Barnetr, S. A. (1963). The rat: A study in behaviour. Chicago: Aldine.

Carder, B., \& Berkowitz, K. (1970). Rat's preference for earned in comparison with free food. Science, 167, 1273-1274.

Herrnste IN, R. J. (1958). Some factors influencing behavior in a two-response situation. Transactions of the New York Academy of Science, 21, 35-45.

Herrnstein, R. J. (1970). On the law of effect. Journal of the Experimental Analysis of Behavior, 13, 243-266.

Hothersale, D., Huey, D., \& Thatcher, K. (1973). The preference of rats for free or response-produced food. Animal Learning \& Behavior, 1, 241-243.

Jensen, G. D. (1963). Preference for bar pressing over "freeloading" as a function of number of rewarded presses. Journal of Experimental Psychology, 65, 451-454.

Mitchell, D. (1976). Experiments on neophobia in wild and laboratory rats: A reevaluation. Journal of Comparative and Physiological Psychology, 90, 190-197.
Mitchell, D., Becnel, J. R., \& Blue, T. (1981). The neophobiaoptimality explanation of contrafreeloading rats: A reassessment. Behavioral and Neural Biology, 32, 454-462.

Mitchell, D., Fish, R. C., \& Calica, D. R. (1982). Rats respond for food in the presence of free food: How free is the "free" food. Learning and Motivation, 13, 257-263.

Mitchell, D., ScotT, D. W., \& Williams, K. D. (1973). Container neophobia and the rat's preference for earned food. Behavioral Biology, 9, 613-624.

Mitcheld, D., Williams, K. D., \& Sutter, J. (1974). Container neophobia as a predictor of preference for earned food by rats. Bulletin of the Psychonomic Society, 4, 182-184.

Neuringer, A. J. (1969). Animals respond for food in the presence of free food. Science, 166, 399-401.

Osborne, S. R. (1977). The free food (contrafreeloading) phenomenon: A review and analysis. Animal Learning \& Behavior, 5, 221-235.

Singr, D. (1970). Preference for bar-pressing to obtain reward over freeloading in rats and children. Journal of Comparative and Physiological Psychology, 73, 320-327.

Tarte, R. D., \& Rasmussen, C. T. (1979). Training effects on contrafreeloading in rats housed in operant chambers. Animal Learning \& Behavior, 7, 69-74.

TARTe, R. D., \& SNyder, R. L. (1972). Barpressing in the presence of free food as a function of food deprivation. Psychonomic Science, 26, 169-170.

TARTE, R. D., \& SNYder, R. L. (1973). Some sources of variation in the bar-pressing versus freeloading phenomenon in rats. Journal of Comparative and Physiological Psychology, 84, 128-133.

Tarte, R. D., \& Vernon, C. (1974). Rat's barpressing in the presence of free food as a function of fixed ratio schedules. Bulletin of the Psychonomic Society, 3, 34-36.

TAYLOR, G. T. (1972). A limitation of the contrafeeding phenomenon. Psychonomic Science, 29, 173-174.

(Manuscript received for publication August 22, 1983.) 